\title{
Use of random PCR (RAPD) technology to analyse phylogenetic relationships in the Lolium/Festuca complex
}

\author{
M. STAMMERS, J. HARRIS, G. M. EVANS, M. D. HAYWARD† \& J. W. FORSTER* \\ Department of Agricultural Sciences, University College of Wales, Aberystwyth, Dyfed SY23 3DD and $\dagger$ Genetics Group, \\ AFRC Institute of Grassland and Environmental Research, Welsh Plant Breeding Station, Aberystwyth, Dyfed SY23 $3 E B$, \\ Wales, U.K.
}

\begin{abstract}
The RAPD PCR technique has been employed to investigate phylogenetic relationships between species of the genera Lolium and Festuca. Several decamer primers were used to generate patterns from groups of genotypes of several different species. The degree of band sharing was used to evaluate genetic distances between species and to construct a phylogenetic tree which is in good overall agreement with classical taxonomy, but contains a number of novel insights. The degree of homoplasy inherent in this approach has been investigated using Southern hybridization. These results are discussed in the context of current work in molecular biosystematics.
\end{abstract}

Keywords: forage grasses, homoplasy, molecular markers, phylogenetic analysis, Southern hybridization, taxonomy.

\section{Introduction}

Some of the most important grasses of temperate agriculture are members of the Lolium and Festuca genera. They typically possess agronomic qualities which make them ideally suited to many livestock farming systems. As both interspecific and intergeneric hybrids can be produced, considerable practical breeding effort has been put into combining their desirable qualities (Thomas \& Humphries, 1991). The Lolium species occur as diploids $(2 n=14)$. Of these, some are inbreeders and predominantly annual, and the others are outbreeders and biennial or perennial (Terrel, 1968). The Festuca species display a wide range of ploidy levels from diploid through to decaploids with the majority being perennial outbreeders. The karyotypes of the complex are based on a fundamental chromosome number of seven (Table 1).

Previous studies of the relationship between the Lolium and Festuca genera used morphological and seed protein analyses (Bulinska-Radomska \& Lester, 1986). These analyses showed a separation of the two genera, but this difference was largely due to the inclusion of the inflorescence data. Seed protein data and

*Correspondence: Institute of Biological Sciences, University of Wales, Aberystwyth, Dyfed SY23 3DD, Wales, U.K. hybridization behaviour suggested a relationship between the Festuca species of section Bovinae (such as $F$. pratensis) and the outbreeding Lolium species. Similarly, Lehvaslaiho et al. (1987), from studies of chloroplast DNA, suggested that $L$. multiflorum is closer to $F$. pratensis and $F$. arundinacea than any of these are to $F$. rubra, a Festuca of the section Ovinae. Borrill (1976) proposed a scheme for the evolution of the Lolium and Festuca species in which the inbreeding Lolium species are derived from ancestral outbreeders whereas the polyploid Festuca species are derived from diploid ancestors which may have resembled $F$. pratensis, with possible contributions from the annual Lolium species in forming the higher polyploids.

As part of our work on the mapping of the Lolium genome (Evans et al., 1991) we became interested in the use of molecular techniques to investigate the phylogenetic relationships among members of the Lolium/Festuca complex. Such studies will be of importance in determining the consequences of interspecific hybridization and introgression events. A number of molecular marker systems are available for such studies, such as variation in chloroplast DNA structure, shared RFLP alleles and the comparison of homologous DNA sequences. In our mapping studies, we have employed a random PCR technique, the RAPD (random amplification of polymorphic DNA) method of Williams et al. (1990). The RAPD method 
Table 1 Members of the Lolium/Festuca complex assessed with reference to taxonomic section, perenniality, breeding system, ploidy level and $C$ value

\begin{tabular}{|c|c|c|c|c|c|}
\hline Species & Section & Perenniality & Breeding system & Ploidy level & 2c DNA (pg) \\
\hline L. perenne & & Perennial & Outbreeding & $2 x=14$ & 4.16 \\
\hline L. multiflorum & & Perennial & Outbreeding & $2 x=14$ & 4.31 \\
\hline L. rigidum & & Annual & Outbreeding & $2 x=14$ & 4.33 \\
\hline L. temulentum & & Annual & Inbreeding & $2 x=14$ & 6.23 \\
\hline L. remotum & & Annual & Inbreeding & $2 x=14$ & 6.04 \\
\hline L. loliaceum & & Annual & Inbreeding & $2 x=14$ & 5.59 \\
\hline F. pratensis & Bovinae & Perennial & Outbreeding & $2 x=14$ & 3.78 \\
\hline F. drymeja & Montanae & Perennial & Outbreeding & $2 x=14$ & 7.17 \\
\hline F. scariosa & Scariosae & Perennial & Outbreeding & $2 x=14$ & 4.73 \\
\hline F. lasto & Montanae & Perennial & Outbreeding & $2 x=14$ & - \\
\hline F. mairei & Scariosae & Perennial & Outbreeding & $4 x=28$ & 6.93 \\
\hline F. appenina & Bovinae & Perennial & Outbreeding & $4 x=28$ & 9.55 \\
\hline F. arund. fenas & Bovinae & Perennial & Outbreeding & $4 x=28$ & - \\
\hline F. arundinacea & Bovinae & Perennial & Outbreeding & $6 x=42$ & 10.53 \\
\hline F. gigantea & Bovinae & Perennial & Facultative inbreeder & $6 x=42$ & 12.82 \\
\hline
\end{tabular}

DNA amounts obtained from Seal (1981).

relies on the use of short (8-10 nucleotide) primers which are annealed to genomic DNA using low temperature conditions. Priming at a number of closely adjacent complementary sites allows the subsequent amplification of dispersed genomic sequences. In $L$. perenne, 6-12 bands are typically produced. Some of the amplified loci are polymorphic and heterozygous, acting as dominant Mendelian alleles. The inheritance of such loci can be scored by following the segregation of the amplified fragment in $F_{2}$ and backcross families. However, many of the RAPD generated fragments, as visualized by agarose gel electrophoresis, are invariant between diverse genotypes of a given species. We have also observed in mapping studies of interspecific crosses that the RAPD generated patterns produced by the same primer with closely related species like $L$. perenne and L. multiflorum showed substantial similarities compared to less closely related species (Stammers, 1992). A similar observation has been made by Caetano-Anolles et al. (1991) using the DAF (DNA amplification fingerprinting) technique, which is similar to the RAPD method but uses shorter primers, lower annealing temperatures and analyses the products using silver staining of polyacrylamide gels. These latter authors have observed both phylogenetically conserved and individual specific bands in their PCR generated patterns. This suggests that the degree of identity between RAPD profiles of related species may be correlated with phylogenetic distance. Demeke et al. (1992) have recently applied the RAPD technique to a study of taxonomic relationships within the Brassica genus, deriving relationships which closely follow the classical pattern for the group.
One problem that may arise in this approach is the phenomenon of homoplasy or convergence, such that bands of similar electrophoretic mobility in RAPD profiles of different species may correspond to nonhomologous DNA sequences. In addition, many RAPD generated amplification products may correspond to repetitive sequences, which are regarded as likely sources of error in phylogenetic studies because of the property of concerted evolution. It would therefore be desirable to determine the presence or absence of homology between shared bands, as well as the repetitive status of the sequences from which they are derived.

This study presents results which suggest that the RAPD technique may be used to obtain diagnostic information to distinguish between different species, and also to obtain phylogenetic information for closely related species.

\section{Materials and methods}

\section{Plant material}

The 14 species examined are described in Table 1. All were obtained from the IGER Genetic Resources Unit except where stated. Lolium temulentum $\mathrm{Ba} 3081$ was collected in Aberystwyth and has been maintained at IGER by selfing over numerous generations. Lolium loliaceum was obtained from the USDA Plant Introduction Service. Lolium rigidum originated from Iran. Three populations of $L$. multiflorum were studied, from Romania, Chaves in North Portugal and the cultivar Tribune, bred by the Welsh Plant Breeding 
Station and incorporating Italian (Po Valley) and North European material. Lolium perenne was obtained from the IGER collection. Festuca pratensis Bf1099.76 was collected from Bergamo, Italy. The $F$. scariosa plants were autotetraploids derived from plants collected in the Sierra Nevada, Spain. Festuca drymeja was obtained from the CSIRO Division of Plant Industry, while $F$. lasto (formerly $F$. boissieri) was collected from Cadiz province, Spain. Tetraploid F. pratensis var. apennina was collected from Genova, Italy. The African species $F$. mairei was obtained from INRA, Clermont-Ferrand, France. Tetraploid $F$. arundinacea subsp. fenas was collected from the French Alps and hexaploid $F$. arundinacea came from the Vaucluse in France. Hexaploid $F$. gigantea was collected from the Tyrol, Austria.

Five plants from each accession were used to obtain RAPD generated patterns to assess levels of intraspecific variation.

\section{DNA extraction}

Plant DNAs were extracted by the CTAB method based on the published protocols of Murray \& Thompson (1980) and Saghai-Maroof et al. (1984).

\section{Polymerase chain reaction}

PCR was performed using a Perkin-Elmer thermocycler. Amplification reactions proceeded in volumes of $50 \mu \mathrm{L}$ under final conditions of $10 \mathrm{~mm}$ Tris- $\mathrm{HCl} \mathrm{pH}$ $8.3,50 \mathrm{mM} \mathrm{KCl}, 2 \mathrm{mM} \mathrm{MgCl}_{2}, 0.001$ per cent (w/v) gelatin, $100 \mu \mathrm{M}$ each of dATP, dGTP, dCTP and TTP, 20 pmoles of primer and $25 \mathrm{ng}$ of template DNA. The reaction mixture was centrifuged briefly and 1 unit of Taq polymerase was added; $50 \mu \mathrm{L}$ of mineral oil was used to overlay the reaction mixture. The cycling regime for the reaction was $94^{\circ} \mathrm{C}$ for $1 \mathrm{~min}, 35^{\circ} \mathrm{C}$ for 1 min and $72^{\circ} \mathrm{C}$ for $2 \mathrm{~min}$, repeated over 45 cycles.

\section{Agarose gel electrophoresis}

Products of the PCR were analysed by electrophoresis in 1.5 per cent agarose gels by overnight runs in electric fields of potential gradient $2 \mathrm{~V} / \mathrm{cm}$. Gels were stained with $0.5 \mu \mathrm{g} / \mathrm{mL}$ ethidium bromide and photographed by transillumination at $313 \mathrm{~nm}$ using Polaroid $665 \mathrm{Pos} /$ Neg film.

\section{Southern blotting and hybridization}

Individual RAPD bands were isolated from agarose gels for labelling by binding to DEAE membrane, followed by elution and purification by phenol:chloroform/chloroform extraction. Probes were labelled using the method of Feinberg \& Vogelstein (1984). Southern blotting was performed by alkaline transfer to Hybond $\mathrm{N}^{+}$(Amersham) as described by Reed (1986). Hybridization and autoradiography protocols were as described by Sandery et al. (1990).

\section{Analysis of phylogenetic data}

RAPD banding profiles were generated as described and the size of bands calculated with reference to known standards. The sizes of bands from each of the five plants from each accession were recorded for each plant $\times$ primer combination, i.e. if a particular band occurred in just one of the five plants scored it was counted as a band on the same basis as a band which may have been present in all five individuals, leading to a superimposition of the patterns. This was performed to avoid the loss of data derived from high frequency polymorphic bands which may be under-represented in the small intraspecific sample size. This means that an independent estimate of intraspecific diversity was not obtained. The banding patterns of each species $\times$ primer combination were then compared within a primer group and the numbers of common bands were recorded in a matrix; this was repeated for each of the three primers. At this point the number of bands found to be in common between each species with all primers was summed and recorded in a matrix. The total numbers of bands produced by the three primers for each plant species were also summed, and the data were used to calculate genetic distance using the formulae of Nei (1987). Firstly, $F$, the proportion of shared fragments was calculated by expressing the total number of shared fragments in relation to the total number of bands produced. The value of $F$ was used to compute $G$, which was in turn used to calculate a measure of genetic distance. The genetic distance data were then analysed using the phylogeny inference package PHYLIP (Felsenstein, 1990). The least squares method of Fitch \& Margoliash (1967) was used to construct a phylogenetic tree.

\section{Results}

Genomic DNA from several different species was used to generate RAPD patterns with a number of oligonucleotide primers in order to identify those primers which would give a large number of bands and hopefully be most useful. Due to logistical constraints, only a small number of primers could be used to generate the basic data set. Three decamer primers were identified: SB3 (5'-GATTCGAGCC-3'), IM5 (5'- 
GAGCTACTGT-3') and IM6 (5'-GGTACAGTCA-3'). These three primers were used to amplify fragments from five different plants from each of the 14 species under study. As shown in Fig. 1, a large number of bands was typically generated with each species. In this case, the decamer primer IM6 was used to generate the patterns. As would be expected, the inbreeding species such as L. temulentum, $L$. loliaceae and the facultative inbreeder $F$. gigantea show very little within-accession variation, whereas the outbreeding species $L$. perenne, L. multiflorum and the remaining Festuca species show considerably more variation. It is clear that each plant species in this study shows a consensus pattern composed of a number of monomorphic bands, usually quite distinct from that of other species, supporting the use of this technique as a diagnostic tool.

Data on band sharing were collected and analysed as described, generating the matrix shown in Table 2, in which values of $F$, the proportion of shared fragments between the species, are shown to the right of the diagonal, and values of $d$, the genetic distance, are shown to the left of the diagonal. Using PHYLIP, the best tree for these data was generated and is shown in Fig. 2. Genetic distances between different populations or species are interpreted by summing the genetic distance values given on the branches of the tree.

To assess the degree of homology between shared bands, a different set of decamer primers was used on a minimal species set. The primers were OPA- $02\left(5^{\prime}\right.$ TGCCGAGCTG-3'), OPA-07 (5'-GAAACGGGTG$\left.3^{\prime}\right)$, OPA-11 (5'-CAATCGCCGT-3') and OPA-18 (5'-AGGTGACCGT-3') whereas the species chosen were two close relatives, $L$. rigidum and $L$. multi- florum, and the more distantly related $F$. gigantea. The three OPA primers were evaluated as part of a related experiment, and it is assumed that an estimate of homoplasy made with these primers will be applicable to RAPD generated patterns in general, including those analysed in the phylogenetic tree construction. RAPD profiles were generated, with a number of unique and shared bands. Six shared bands were selected and used as hybridization probes to the profiles from which they were derived (Fig. 3a-f). The results show that the majority (four of six) of the selected fragments show sequence homology to the shared band or bands in other species.

\section{Discussion}

The Lolium species are all grouped together on the phylogenetic tree generated by the RAPD study. The three $L$. multiflorum accessions and $L$. perenne are clustered together, with the annual species placed more distant from this grouping. Naylor (1960) observed that $L$. perenne and $L$. multiflorum cross reciprocally as readily as two populations or varieties of the same species. The clustering of $L$. multiflorum and $L$. perenne on the tree supports the view that they are very closely related, although the Portuguese $L$. multiflorum from Chaves is detached from the other $L$. multiflorum accessions. This line has been observed to be rather more annual than the other $L$. multiflorum populations, perhaps indicating some introgression from the annual outbreeder L. rigidum (G. M. Evans, personal communication).

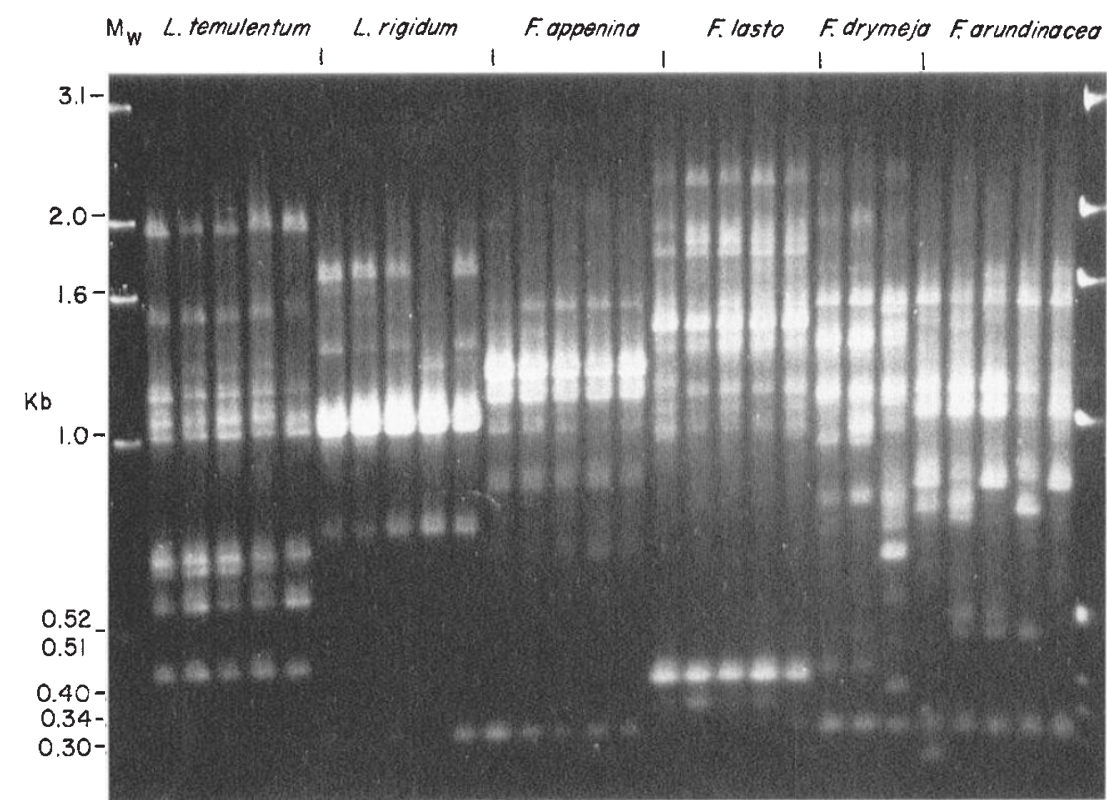

Fig. 1 RAPD amplification profiles generated by the use of primer IM6 on a number of individuals from a range of Lolium and Festuca species. The amplification products were visualized following agarose gel electrophoresis on 1.5 per cent gels. Molecular weights of standards are indicated in kilobases. 
Table 2 Matrix showing values of $F$ (the proportion of shared fragments) to the right of the diagonal, and values of $d$ (the genetic distance) to the left of the diagonal

\begin{tabular}{|c|c|c|c|c|c|c|c|c|c|c|c|c|c|c|c|c|c|}
\hline & 1 & 2 & 3 & 4 & 5 & 6 & 7 & 8 & 9 & 10 & 11 & 12 & 13 & 14 & 15 & 16 & $\begin{array}{l}\text { Total no. } \\
\text { of bands }\end{array}$ \\
\hline 1 & - & 0.239 & 0.346 & 0.308 & 0.217 & 0.476 & 0.275 & 0.286 & 0.337 & 0.087 & 0.303 & 0.081 & 0.174 & 0.103 & 0.215 & 0.167 & 38 \\
\hline 2 & 0.053 & - & 0.278 & 0.195 & 0.297 & 0.247 & 0.197 & 0.293 & 0.081 & 0.133 & 0.229 & 0.215 & 0.167 & 0.174 & 0.107 & 0.191 & 29 \\
\hline 3 & 0.038 & 0.047 & - & 0.229 & 0.409 & 0.426 & 0.235 & 0.313 & 0.205 & 0.189 & 0.143 & 0.205 & 0.243 & 0.217 & 0.143 & 0.153 & 43 \\
\hline 4 & 0.043 & 0.061 & 0.054 & - & 0.408 & 0.165 & 0.316 & 0.396 & 0.225 & 0.095 & 0.192 & 0.202 & 0.191 & 0.194 & 0.250 & 0.253 & 53 \\
\hline 5 & 0.056 & 0.044 & 0.032 & 0.032 & - & 0.225 & 0.322 & 0.347 & 0.244 & 0.312 & 0.116 & 0.074 & 0.105 & 0.188 & 0.167 & 0.263 & 45 \\
\hline 6 & 0.026 & 0.051 & 0.030 & 0.068 & 0.055 & - & 0.163 & 0.247 & 0.180 & 0.160 & 0.232 & 0.175 & 0.080 & 0.143 & 0.085 & 0.154 & 44 \\
\hline 7 & 0.047 & 0.060 & 0.053 & 0.042 & 0.041 & 0.068 & - & 0.295 & 0.253 & 0.137 & 0.120 & 0.180 & 0.096 & 0.220 & 0.232 & 0.237 & 42 \\
\hline 8 & 0.046 & 0.045 & 0.042 & 0.033 & 0.038 & 0.051 & 0.044 & - & 0.184 & 0.167 & 0.165 & 0.247 & 0.262 & 0.237 & 0.275 & 0.223 & 53 \\
\hline 9 & 0.039 & 0.097 & 0.059 & 0.055 & 0.052 & 0.064 & 0.050 & 0.063 & - & 0.263 & 0.163 & 0.222 & 0.290 & 0.141 & 0.194 & 0.203 & 45 \\
\hline 10 & 0.094 & 0.076 & 0.062 & 0.090 & 0.077 & 0.067 & 0.075 & 0.067 & 0.049 & - & 0.139 & 0.119 & 0.226 & 0.141 & 0.035 & 0.185 & 31 \\
\hline 11 & 0.059 & 0.054 & 0.073 & 0.061 & 0.082 & 0.054 & 0.080 & 0.068 & 0.068 & 0.075 & - & 0.286 & 0.222 & 0.124 & 0.059 & 0.051 & 41 \\
\hline 12 & 0.097 & 0.057 & 0.059 & 0.059 & 0.101 & 0.065 & 0.064 & 0.051 & 0.056 & 0.081 & 0.046 & - & 0.149 & 0.237 & 0.191 & 0.086 & 36 \\
\hline 13 & 0.065 & 0.067 & 0.052 & 0.062 & 0.086 & 0.098 & 0.090 & 0.049 & 0.045 & 0.055 & 0.055 & 0.072 & - & 0.197 & 0.379 & 0.215 & 31 \\
\hline 14 & 0087 & 0.065 & 0.056 & 0.061 & 0.062 & 0.073 & 0.056 & 0.053 & 0.074 & 0.074 & 0.079 & 0.053 & 0.060 & - & 0.119 & 0.189 & 40 \\
\hline 15 & 0.057 & 0.085 & 0.073 & 0.051 & 0.067 & 0.095 & 0.054 & 0.047 & 0.061 & 0.134 & 0.111 & 0.062 & 0.035 & 0.081 & - & 0.131 & 27 \\
\hline 16 & 0.067 & 0.062 & 0.070 & 0.050 & 0.049 & 0.070 & 0.053 & 0.054 & 0.059 & 0.063 & 0.117 & 0.095 & 0.057 & 0.062 & 0.077 & - & 31 \\
\hline
\end{tabular}

1: L. temulentum; 2: L. loliaceum; 3: L. rigidum; 4: L. multiflonum Tribune; 5: L. multiflorum Romania; 6: L. multiflorum Chaves; 7: L. perenne; 8: F. pratensis; 9: F. drymeja; 10: F. lasto; 11: F. scariosa; 12: F. mairei; 13: F. appenina; 14: F. arundinacea ssp. fenas; 15: F. gigantea; 16: F, arundinacea.

Fig. 2 Phylogenetic tree of the Lolium/ Festuca complex. Genetic distances $(d)$ are indicated on each arm of the tree.

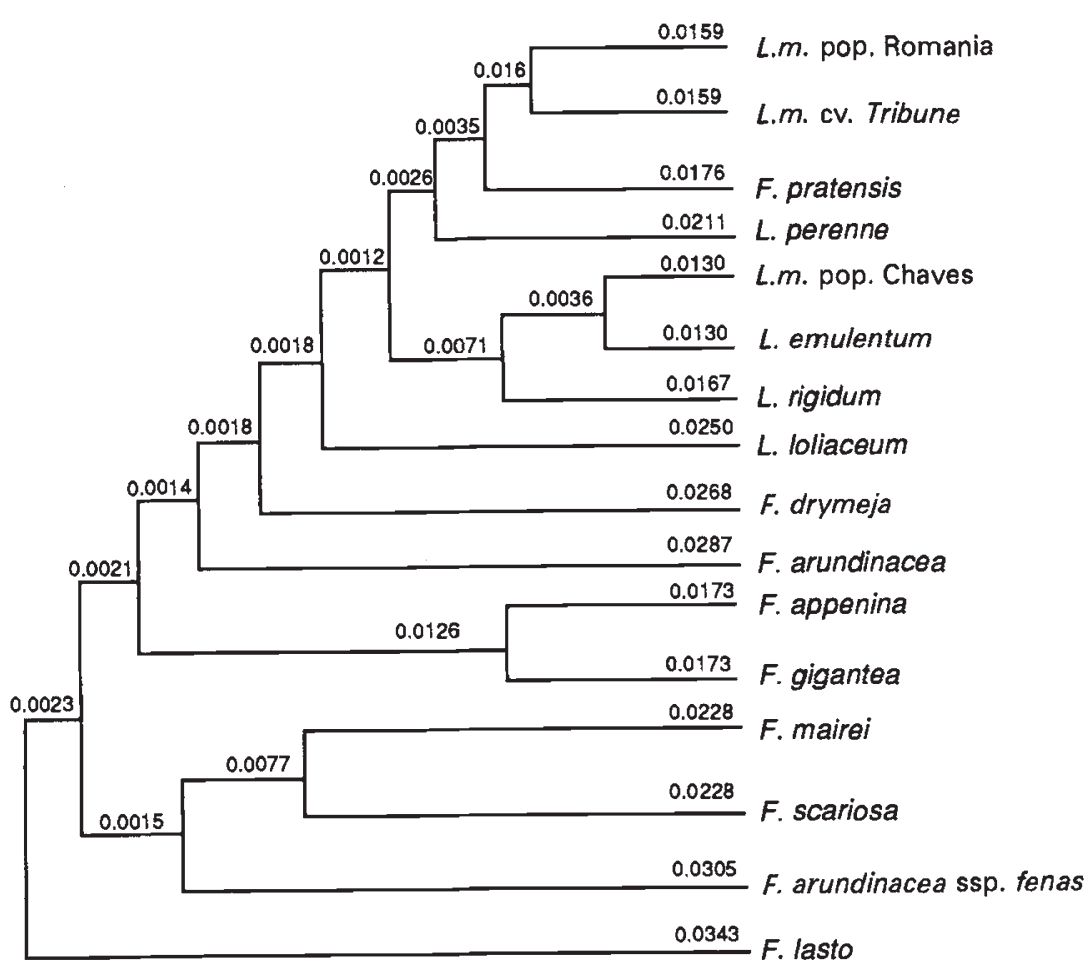

L.m. $=$ Lolium multiflorum,

(not to scale) 

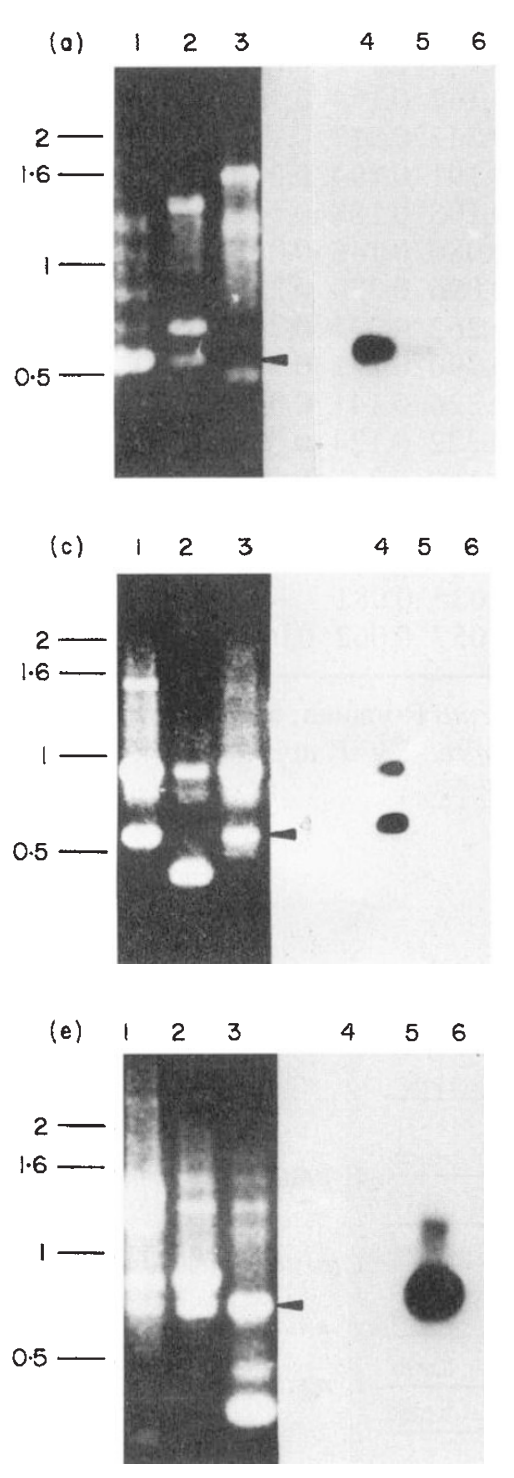
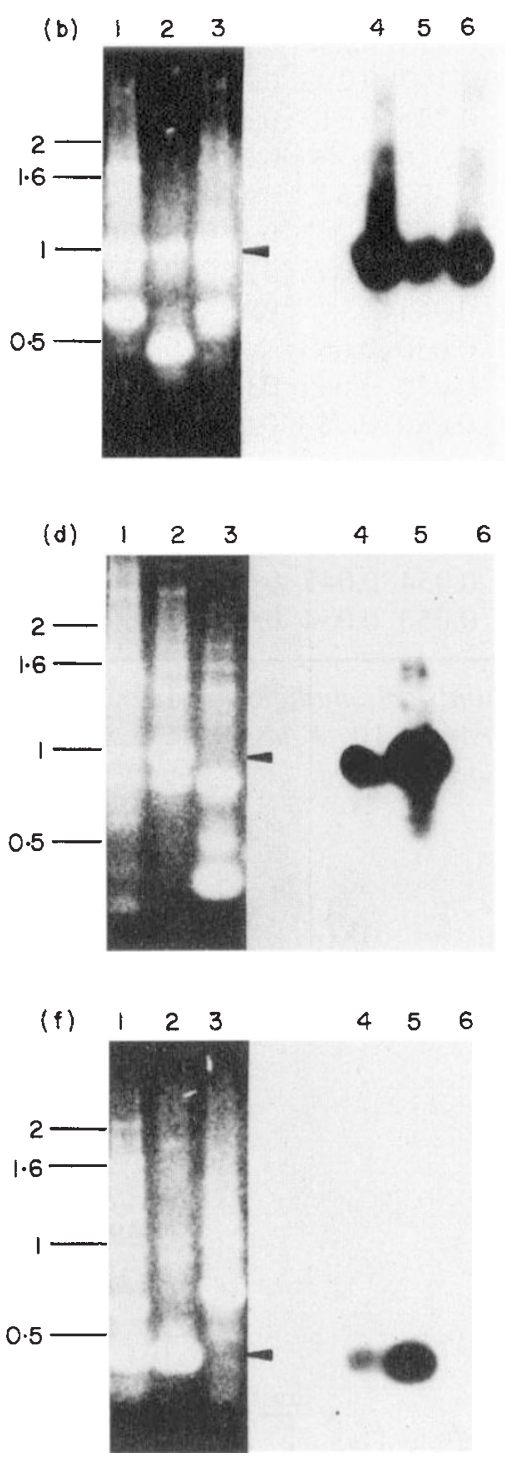

Fig. 3 Electrophoretograms and corresponding Southern hybridization autoradiographs demonstrating RAPD band homology between Lolium and Festuca species. Molecular weights of standards are indicated in kilobases. (a) Lanes 1-3 show the RAPD profile generated by the primer OPA- 02 using the following templates: lane $1, L$. rigidum; lane 2, L. multiflorum; lane $3, F$. gigantea. Lanes 4-6 show the results of hybridization of the purified $0.5 \mathrm{~kb} L$. rigidum fragment which is shared with L. multiflorum. (b) Lanes 1-3 show the RAPD profile generated by the primer OPA-07 using the same templates as in Fig. 3(a). Lanes 4-6 show the results of the hybridization of the $0.9 \mathrm{~kb} \mathrm{~L}$. rigidum fragment which is shared with $L$. multiflorum and $F$. gigantea. (c) Lanes 1-3 show the RAPD profile generated by the primer OPA-07 using the same templates as in Fig. 3(a). Lanes 4-6 show the results of the hybridization of the $0.6 \mathrm{~kb} L$. rigidum fragment which is shared with $F$. gigantea. (d) Lanes 1-3 show the RAPD profile generated by the primer OPA- 11 using the same templates as in Fig. 3(a). Lanes 4-6 show the results of the hybridization of the $0.8 \mathrm{~kb}$ L. multiflorum fragment which is shared with $F$. gigantea. (e) Lanes 1-3 show the RAPD profile generated by the primer OPA- 11 using the same templates as in Fig. 3(a). Lanes 4-6 show the results of the hybridization of the $0.7 \mathrm{~kb}$ L. multiflorum fragment which is shared with $F$. gigantea. (f) Lanes 1-3 show the RAPD profile generated by the primer OPA- 18 using the same templates as in Fig. 3(a). Lanes 4-6 show the results of the hybridization of the $0.3 \mathrm{~kb}$ L. multiflorum fragment which is shared with $L$. rigidum.
One of the most immediately striking features of the tree is the placement of $F$. pratensis among the members of the Lolium genus. In fact, this observation is consistent with some previous studies. Jauhar (1976) showed that hybridization between $L$. perenne and $F$. pratensis or L. multiflorum and $F$. pratensis occurred easily with either species as male or female parent, giving mostly seven pairs of bivalents at meiosis in the hybrids. It was suggested that there is a very close phylogenetic proximity between these three species and $F$. pratensis is not sufficiently distinct to deserve a specific rank in a genus separate from $L$. perenne and
L. multiflonum. Studies of the distribution of repetitive DNA sequences derived from L. perenne (J. W. Forster, unpublished data) also indicate a close relationship between $F$. pratensis and the outbreeding Lolium species.

The polyploid Festuca species are thought to have arisen as a result of various hybridization events between the different diploid Festuca and perhaps even Lolium species. A contribution from $F$. pratensis has been suspected for the majority of the polyploid species, especially those of the Bovinae section. There is also evidence for the involvement of $F$. scariosa in 
certain polyploid species of the Scariosae and Bovinae sections (Borrill, 1972). Borrill et al. (1977) demonstrated genetic affinity between $F$. scariosa and two diploid species of the Montanae section, $F$. donax and F. drymeja. In a morphological study BulinskaRadomska \& Lester (1986) also found these species to be related, and further suggested, from studying seed protein profiles, that $F$. scariosa along with one of the diploid species of section Montanae were involved in the origin of tetraploid $F$. mairei of section Scariosae. Bulinska-Radomska \& Lester (1986) also believe $F$. mairei to be one of the key species in the evolution of the higher polyploids such as $F$. arundinacea. This close relationship of $F$. scariosa and $F$. mairei is reflected also in its position in the RAPD generated tree, $F$. scarios $a$ and $F$. mairei being situated together. Similarly, the allotetraploid $F$. arundinacea subsp. fenas is situated close to $F$. mairei and $F$. scariosa, although they are morphologically dissimilar. Studies of chromosome behaviour in hybrids of $F$. mairei and $F$. arundinacea subsp. fenas by Malik \& Thomas (1967) demonstrated the presence of similar genomes in these two species, showing again the strong correspondence between classical and RAPD based taxonomy.

However, some anomalies can be observed in the order of the Festuca species, in particular the relationship of $F$. drymeja and $F$. lasto. These are considered to be taxonomically quite similar yet they are separated by a relatively large distance in the phylogram. The tetraploid $F$. pratensis subsp. apennina is placed some distance from its diploid counterpart $F$. pratensis subsp. pratensis. In this case, however, they are morphologically dissimilar and hence the conventional classification may be questionable.

A noticeable feature of the tree is that the values for genetic distance are all very close. This is because the analysis used did not take into account the genetic divergence within the population samples of five plants. It would be expected that consideration of withinpopulation divergence for the three accessions of $L$. multiflorum would lead to a reduction of the genetic distances between them, making the values for genetic distances between species appear larger by comparison.

The RAPD generated phylogenetic tree shows a number of strong similarities to those generated by previous studies, along with a number of anomalies which can be clearly rationalized in the light of current knowledge. The number of possible trees which were examined to select the most likely tree was 1227 , and it is hardly likely that such a plausible tree would have arisen at random. However, there are a number of possible causes for concern in the use of RAPD generated patterns for phylogenetic inference. The main problems concern: (i) the extent to which it is possible to infer homology of bands showing the same rates of migration in the electrophoretic system; (ii) the causes of variation in the fragment mobility; and (iii) the origin and repetitive status of the sequences under analysis.

Knowing the identity of shared fragments is essential because a successful analysis depends on these fragments originating from amplification of the same sequence. The validity of this assumption, that fragments of similar mobility derive from homologous DNA sequences, has been considered for restriction endonuclease based analysis, i.e. the presence of shared RFLP alleles. The occurrence of similar-sized fragments of different origin has been termed convergence. It may be that convergence is likely to be inherent in the RAPD technique, as the size range of bands amplified and resolved is relatively small, from $100 \mathrm{bp}$ to 2.5 $\mathrm{kbp}$. We have examined the homology between individual RAPD bands of shared size by hybridization of Southern blots of RAPD profiles. In the small sample of bands which has been analysed, two-thirds (four of six) showed homology. Three of these positive results were revealed by bands shared between the two close relatives $L$. rigidum and $L$. multiflorum, while the fourth is shared by all three species. A higher degree of conservation would be expected between the two Lolium species compared to the Festuca outgroup. A fifth band shared by $L$. multiflorum and $F$. gigantea gave a simple negative result, and the sixth assayed band produced an unusual hybridization pattern to several bands in the RAPD profile. Clearly a substantial proportion of the RAPD bands corresponds to homologous sequences with conserved organization.

A consideration of the causes of change in fragment migration is also important. The RAPD method involves the annealing of decamer primers under relaxed conditions. The annealing of primers to a specific sequence is thought to be more critical at the $3^{\prime}$ end of the primer than at the $5^{\prime}$ end, so only base substitution changes near the $3^{\prime}$ end of the primer-template complex are likely to alter significantly the efficiency of priming. It is more likely that insertion/deletion events are important in generating variation in RAPD patterns. The analysis based on genetic distance may not be appropriate where such structural variation occurs (Dowling et al., 1990). However, a similar approach was used in a phylogenetic study of the Lycopersicon genus by Miller \& Tanksley (1990), using RFLP data. Here it was recognized that the majority of RFLP was attributable to DNA rearrangements rather than to point mutations. The RFLP generated dendrogram, however, was consistent with previous classification based on morphology and crossability studies. This implies that the DNA-level varia- 
tion from rearrangement events in plants may be amenable to analysis using the equations of Nei (1987).

Lastly, the nature of the sequences detected by the RAPD analysis is of importance. It is clear that a substantial number of the amplified fragments contain repetitive domains, as indicated by their behaviour when used as hybridization probes (M. Stammers, J. A. K. Will \& J. W. Forster, unpublished data). In this analysis, the sequences used as probes in Fig. 3(e,f) seem to correspond to middle repetitive sequences, as they detect hybridization smears with superimposed discrete bands (data not shown). It has been suggested that repetitive sequences are not suitable for use in phylogenetic analysis because they are prone to homogenization events leading to concerted evolution. This may lead to discrepancies in the phylogenetic tree because of a biased sequence convergence resulting from the rapid spread of a variant repeated sequence. Two repetitive DNA families derived from $L$. perenne have been examined (J. W. Forster, unpublished data) and shown to have common structures among members of both genera. Hence a similarity in genomic architecture may tend to reduce any confounding effects due to the use of repetitive sequences.

One further unusual result of this study is the failure to observe an increase in pattern complexity commensurate with the nuclear DNA content of the species under investigation. This applies to the polyploid Festuca species shown in Table 1, which have C-values in general higher than the diploid species. We have performed RAPD analysis on a range of plant and fungal species and have found RAPD patterns of similar complexity over a wide range of $\mathrm{C}$-values. The origin of this effect is unknown.

Although there are a number of problems concerning the theoretical basis of RAPD phylogeny analysis, along with specific problems due to polyploidy and intraspecific variation in the Lolium/Festuca complex, there are also some clear advantages. Many loci are examined at the same time, which is important because the divergences of many different loci are measured together and thus will tend to minimize the contribution of those loci which are under selection pressure. Hence it is possible that analysis of randomly amplified fragments from diverse regions of the genome will give data on the divergence of the genome as a whole. The RAPD phylogeny approach is only likely to be appropriate for closely related groups, producing uprooted phylograms. This is well exemplified in the case of the Lolium/Festuca complex, where other classical and molecular data suggest a close relationship.

\section{Acknowledgements}

This work was supported by AFRC Link research grant LRG 153. We thank Dr Jacqueline Will and Professor R. N. Jones for helpful discussions and Ms Kerry Stoker for the preparation of the figures.

\section{References}

BORRILL, м. 1972. Studies in Festuca. III. The contribution of $F$. scariosa to the evolution of the polyploids in sections Bovinae and Scariosae. New Phytol., 71, 523-532.

BORRILL, M. 1976. Temperate grasses. In: Simmons, N. W. (ed.) Evolution of Crop Plants, pp. 137-142. Longman, London.

BORRILL, M., KIRBY, M. AND MORGAN, G. M. 1977. Studies in Festuca. II. Interrelationship of some putative diploid ancestors of the polyploid broad-leaved fescues. New Phytol., 78, 661-674.

BULINSKA-RADOMSKA, Z. AND LESTER, R. N. 1986. Intergeneric relationships of Lolium, Festuca and Vulpia (Poaceae) and their phylogeny. Plant Syst. Evol., 159, 217-227.

CAETANO-ANOLLES, G., BASSAM, B. J. AND GRESSHOFF, P. M. 1991. DNA amplification fingerprinting using very short arbitrary oligonucleotide primers. Bio/Technology, 9, 553-556.

DEMEKe, T., ADAMS, R. P. AND CHBbBAR, R. 1992. Potential taxonomic use of random amplified polymorphic DNA (RAPD): a case history in Brassica. Theor. Appl. Genet., 84, 990-994.

DOWLING, T. E., MORITZ, C. AND PALMER, J. D. 1990. Nucleic acids: restriction site analysis. In: Hillis, D. M. and Moritz, C. (eds) Molecular Systematics, pp. 250-318. Sinauer Associates, Sunderland, MA.

EVANS, G. M., HAYWARD, M. D., FORSTER, J. W., MCADAM, N. J., SCANLON, M. J., STAMMERS, M. AND WILL, J. A. K. 1991. Genome analysis and its manipulation in Lolium. In: De Nijs, A. P. M. and Elgersma, A. (eds) Fodder Crops Breeding: Achievements, Novel Strategies and Biotechnology. Proceedings of the 16th meeting of the fodder crops section of Eucarpia, Wageningen, Netherlands, 18-22 November 1990, pp. 141-146. Pudoc, Wageningen.

FEINBERG, A. P. AND Vogelstein, A. P. 1984. A technique for radiolabelling DNA restriction fragments to high specific activity (Addendum). Analyt. Biochem., 137, 266-267.

FELSENSTEIN, J. 1990. PHYLIP manual version 3.3. University Herbarium, University of California, Berkeley, CA.

FITCH, W. M. AND MARGOLIASH, E. 1967. Construction of phylogenetic trees. Science, 155, 279-284.

JAUHAR, P. P. 1976. Chromosome pairing in some triploid and trispecific hybrids in Lolium/Festuca and its phylogenetic implications. Chromosomes Today, 5, 165-177.

LEHVASLAiHO, H., SAURA, A. AND LOKKI, J. 1987. Chloroplast DNA variation in the grass tribe Festucae. Theor. Appl. Genet., 74, 298-302.

MALIK, C. P. AND THOMAS, P. T. 1967. Cytological relationships and genome structure of some Festuca species. Caryologia, 209, 1-39. 
MILLER, J. C. AND TANKSLEY, S. D. 1990. RFLP analysis of phylogenetic relationships and genetic variation in the genus Lycopersicon. Theor. Appl. Genet., 80, 437-448.

MURRAY, M. G. AND THOMPSON, W. F. 1980. Rapid isolation of high molecular weight plant DNA. Nucl. Acids Res., 8, 4321.

NAYLOR, B. 1960. Species differentiation in genus Lolium. Heredity, 15, 219-223.

NEI, M. 1987. Molecular Evolutionary Genetics. Columbia University Press, New York.

REED, K. C. 1986. Alkaline Southern blotting to Zetaprobe membrane. Bio-Rad Bulletin, no. 1233.

SAGHAI-MAROOF, M. A., SOlMAN, K. M., JORGENSEN, R. A. AND ALLARD, R. W. 1984. Ribosomal DNA spacer length polymorphisms in barley: Mendelian inheritance, chromosome location and population dynamics. Proc. Natl. Acad. Sci. U.S.A., 81, 8014-8018.
SANDERY, M. J., FORSTER, J. W., BLUNDEN, R. AND JONES, R. N. 1990. Identification of a family of repeated sequences on the rye B-chromosome. Genome, 33, 908-913.

SEAL, A. G. 1981. Cytogenetic Studies in Festuca and Lolium. Ph.D. Thesis, University of Wales.

STAMMERS, M. 1992. Molecular Mapping and Biosystematics of the Festuceae. Ph.D. Thesis, University of Wales.

TERREL, E. E. 1968. A taxonomic revision of the genus Lolium. U.S. A.R.S. Techn. Bull., 1392, 1-65.

THOMAS, H. AND hUMPHREYS, M. O. 1991. Progress and potential of interspecific hybrids of Lolium and Festuca. J. Agric. Sci. Camb., 117, 1-8.

WILLIAMS, J. G. K., KUBELIK, A. R., LIVAK, K. J., RAFALSKI, J. A. AND TINGEY, S. V. 1990. DNA polymorphisms amplified by arbitrary primers are useful as genetic markers. $\mathrm{Nucl}$. Acids Res., 18, 6531-6535. 